\title{
On Young towers associated with infinite measure preserving transformations
}

\author{
H. Bruin, M. Nicol† D. Terhesiu
}

October 16, 2009

\begin{abstract}
For a $\sigma$-finite measure preserving dynamical system $(X, \mu, T)$, we formulate necessary and sufficient conditions for a Young tower $(\Delta, \nu, F)$ to be a (measure theoretic) extension of the original system. Because $F$ is pointwise dual ergodic by construction, one immediate consequence of these conditions is that the Darling Kac theorem carries over from $F$ to $T$. One advantage of the Darling Kac theorem in terms of Young towers is that sufficient conditions can be read off from the tail behaviour and we illustrate this with relevant examples. Furthermore, any two Young towers with a common factor $T$, have return time distributions with tails of the same order.
\end{abstract}

\section{Introduction}

If $T$ is a conservative, ergodic measure preserving transformation (c.e.m.p.t.) of an infinite measure space $(X, \mathcal{B}, \mu)$, then Birkhoff's Ergodic Theorem is not very informative about the asymptotic behavior of the ergodic sums $S_{n}(f)=\sum_{k=0}^{n-1} f \circ T^{k}$ since, in contrast to the finite measure case, for all $f \in L^{1}(\mu)$

$$
\frac{S_{n}(f)}{n} \rightarrow 0 \quad \mu \text {-a.e. } \quad \text { as } n \rightarrow \infty .
$$

In fact, as proved by Aaronson in [1, Theorem 2.4.2], for an infinite c.e.m.p.t. of $(X, \mathcal{B}, \mu)$ there are no constants $c_{n}>0$ such that for all $f \in L^{1}(\mu)$

$$
\frac{S_{n}(f)}{c_{n}} \rightarrow \int_{X} f d \mu \quad \mu \text {-a.e. } \quad \text { as } n \rightarrow \infty .
$$

Still, for certain infinite measure preserving transformations $T$ of $(X, \mathcal{B}, \mu)$, there exist constants $a_{n}$ such that for all $f \in L^{1}(\mu), a_{n}^{-1} S_{n}(f)$ converges in distribution to a non-trivial limit (see $\S 3.2-\S 3.6$ in [1] for a description of the general setting and examples).

Representative systems of this kind have been found within the class of infinite measure preserving transformations with a finite number of indifferent fixed points

${ }^{*}$ The support of EPSRC grant EP/F037112/1 is gratefully acknowledged, and also the hospitality of Delft University of Technology

${ }^{\dagger}$ MN gratefully acknowledges the support of NSF grants DMS 0600927 and DMS-0607345

¥DT thanks EPRS and MASCOS at UNSW and EPSRC grant EP/F031807/1 for financial support and the University of Houston for hospitality. 
or orbits (see [1, Chapter 4] and references therein; see also [3, 19, 20, 23, 24, 16, $13,12,9]$ ). A standard example is the Pomeau-Manneville (PM) map. For a fixed $\alpha \geq 0$, this map is given by

$$
T:[0,1] \circlearrowleft, \quad T(x)=x+x^{1+\alpha} \quad(\bmod 1),
$$

and it has an indifferent fixed point at 0 . It is well known that $T$ admits a unique absolutely continuous invariant measure (a.c.i.m.) $\mu \ll m$ ( $m$ is Lebesgue) which is finite for $\alpha<1$ and infinite for $\alpha \geq 1$. In the works cited above, it has been shown that a distributional limit theorem (of Darling Kac type) holds for PM maps for all $\alpha \geq 1$. At the threshold value $\alpha=1$ (called the barely infinite measure case in [3]) it gives a weak law. In probabilistic terms this is a weak law of large numbers for $\alpha=1$ : for all $f \in L^{1}(\mu)$ with $\mu(f) \neq 0$ and every $\epsilon>0$

$$
\lim _{n \rightarrow \infty} \nu\left(A \cap\left\{x:\left|a_{n}^{-1} S_{n}(f)(x)-\mu(f)\right| \geq \epsilon\right\}\right)=0,
$$

where $a_{n}=n / \log n, \nu$ is any probability measure $\nu \ll \mu$ and $A \in \mathcal{B}([0,1])$ with $0<\mu(A)<\infty$. Distributional limit theorems for transformations similar to the one above have been generalized to certain infinite measure sets at the threshold value $\alpha=1$ in $[3,16]$. Under further conditions, distributional limit theorems for the occupation times of infinite measure sets associated with transformations similar to the one above have been obtained in $[3,16,24]$.

In this work we model infinite measure preserving transformations via Young towers, derive a new version of the Darling Kac (DK) theorem and argue for its efficient application in some particular examples.

Acknowledgement: We would like to thank the referee whose comments have helped to improve the presentation of this paper considerably. We also like to thank Omri Sarig and Roland Zweimüller for useful discussions on related topics.

\section{Main results and background review}

The study of the ergodic properties of dynamical systems by means of induced transformations and tower (sky-scraper) constructions goes back to Renyi, Kakutani and Rohlin. Originally these were formulated for first return maps $T_{E}(x)=T^{\varphi_{E}(x)}(x)$ and first return times $\varphi_{E}(x)=\min \left\{j \geq 1: T^{j}(x) \in E\right\}$, whenever defined. However, return times $R$ that are not necessarily first return times can be used as well, and makes the method more widely applicable. Young in $[17,18]$ gave an axiomatic approach, introducing conditions on distortion in terms of separation times, see (YT6) below. It is her approach that we will follow in this paper, see Section 3 for details. Our main aim in this work is to formulate necessary and sufficient conditions that ensure that the invariant measure of the tower system projects to the $\sigma$-finite invariant measure of the original system. Building on the results of Zweimüller [21] we show the following:

Theorem 2.1. Let $T$ be a non-singular, ergodic transformation of $(X, \mathcal{B}, m)$ with a $\sigma$-finite invariant measure $\mu \ll m$. Suppose that $T$ has a Young tower description $\left(\Delta, \mathcal{B}(\Delta), F, m^{\prime}\right)$ with base map $\left(\Delta_{0}, T^{R}, m_{0}\right)$ and $\int R d m_{0}=\infty$ and factor map $\pi: \Delta \rightarrow X$. Furthermore, let $\nu \ll m^{\prime}$ be an $F$-invariant measure.

Then $\mu=\pi_{*} \nu$ (up to a multiplicative constant) if and only if

$$
\begin{aligned}
& \text { for every set } E \subseteq \Delta_{0}, 0<\pi_{*} \nu(E)<\infty \text { and for inducing time } \\
& \tau: E \rightarrow \mathbb{N} \text { given by }\left(T^{R}\right)_{E}=\left(T_{E}\right)^{\tau} \text { we have } \int_{E} \tau d m<\infty .
\end{aligned}
$$


We emphasize that $T_{E}$ and $\left(T^{R}\right)_{E}$ are the first return maps to $E$ under $T$ and $T^{R}$ respectively; if $T^{R}$ happens to be the first return map to $\Delta_{0}$, then $\tau \equiv 1$ and (2) holds trivially. If a YT satisfying (2) is found, it is immediate that $F$ and hence $T$ is pointwise dual ergodic and $\Delta_{0}$ is a Darling Kac set for the Young tower $(\Delta, F)$, see Lemma 4.5. To state further consequences of the above theorem, we first recall some important tools of infinite ergodic theory.

\subsection{Prerequisites from infinite ergodic theory}

Notation. We write $a_{n} \sim b_{n}$ if $\frac{a_{n}}{b_{n}} \rightarrow 1$ as $n \rightarrow \infty$ and $a_{n} \propto b_{n}$ if $\frac{a_{n}}{b_{n}} \rightarrow c$ as $n \rightarrow \infty$ for some $0<c<\infty$. In the latter case we call the sequences $\left(a_{n}\right)$ and $\left(b_{n}\right)$ asymptotically equivalent. Infinite $\sigma$-finite measures are only determined up to a positive multiplicative constant, and this means that some limits are only taken up to a multiplicative constant, see e.g. Remark 2.2 and Proposition 2.5 below.

A function $a:\left(c_{0}, \infty\right) \rightarrow(0, \infty)$ (or a sequence interpreted as $\left.t \mapsto a_{[t]}\right)$ is slowly varying at $\infty$ if $a$ is Borel measurable and $\frac{a(c t)}{a(t)} \rightarrow 1$ as $t \rightarrow \infty$. A function $b$ : $\left(c_{0}, \infty\right) \rightarrow(0, \infty)$ is regularly varying at $\infty$ with index $\gamma$, denoted as $b \in \mathcal{R}_{\gamma}$, if $b(t)=t^{\gamma} a(t)$ with $a(t)$ slowly varying at infinity.

When $T$ is a c.e.m.p.t. of the ( $\sigma$-finite) infinite measure space $(X, \mathcal{B}, \mu)$, distributional characterizations of $T$ are often given in terms of a reference set $Y$ of finite measure, for instance Darling Kac type theorems in [1, 16, 23]. Essentially, $Y$ is a candidate for being a suitable reference set if its wandering rate

$$
w_{n}(Y):=\mu\left(\cup_{k=0}^{n-1} T^{-k} Y\right)=\sum_{k=0}^{n-1} \mu\left(Y \cap\left\{\varphi_{Y}>k\right\}\right)
$$

belongs to $\mathcal{R}_{\gamma}$ for some $\gamma \in[0,1]$ (see for instance $[3,16,23]$ ).

The wandering rate $\left(w_{n}(Y)\right)$ depends on the set $Y$. However, for some transformations $T$ of $(X, \mathcal{B}, \mu)$ there are sets $A, 0<\mu(A)<\infty$ of minimal wandering rate in the sense that for all $B \in \mathcal{B}, 0<\mu(B)<\infty$, we have $\liminf _{n \rightarrow \infty} w_{n}(B) / w_{n}(A) \geq 1$. In this situation $w_{n}(A)$ is a characteristic of the system $(X, T, \mu)$; is called the wandering rate of the system and denoted as $w_{n}(T)$. One such situation is given by pointwise dual ergodic (p.d.e.) transformations, see $\S 3$ in [1]. A conservative ergodic measure preserving transformation $T$ of $(X, \mathcal{B}, \mu)$ is called pointwise dual ergodic if there is a positive sequence $\left\{a_{n}(T)\right\}_{n \geq 1}$, called a return sequence, such that for all $f \in L^{1}(\mu)$

$$
\frac{1}{a_{n}(T)} \sum_{k=0}^{n-1} \hat{\mathbf{T}}^{k}(f) \rightarrow \int_{X} f d \mu, \mu \text {-a.e. as } n \rightarrow \infty .
$$

Here $\hat{\mathbf{T}}: L^{1}(\mu) \circlearrowleft$ is the associated dual operator defined by

$$
\int_{X} \hat{\mathbf{T}} f \cdot g d \mu=\int_{X} f \cdot(g \circ T) d \mu
$$

for $f \in L^{1}(\mu)$ and $g \in L^{\infty}(\mu)$. Many proofs in the literature on pointwise ergodic duality require that $\int_{X} f d \mu>0$, and we will make this assumption throughout this paper.

Remark 2.2. As a consequence of [1, Theorem 3.3.1 and Proposition 3.7.1] for a p.d.e. transformation, the sequence $a_{n}(T)$ in (3) above is uniquely determined up to asymptotic equivalence, just as the $\sigma$-finite measure is determined up to a 
multiplicative constant. Once matching choices of $a_{n}(T)$ and $\mu$ are made, (3) holds for all $f \in L^{1}(\mu)$.

The set $A \in \mathcal{B}, 0<\mu(A)<\infty$ is a Darling Kac set for $T$ if the convergence in (3) takes place uniformly almost everywhere on $A$ for the indicator function $f=1_{A}$.

The Darling-Kac (DK) property is directly linked to pointwise dual ergodicity. More precisely, according to [1, Proposition 3.7.5], if $T$ is a c.e.m.p.t. of $(X, \mathcal{B}, \mu)$ and $T$ has a Darling-Kac set, then $T$ is pointwise dual ergodic.

Both the return sequence and the wandering rate of a p.d.e. transformation can be obtained directly by estimating these quantities for its corresponding measure theoretic extensions.

Definition 2.3. Given that $\left(X^{\prime}, \mathcal{B}^{\prime}, \mu^{\prime}, T^{\prime}\right)$ and $(X, \mathcal{B}, \mu, T)$ are measure preserving transformations, $T^{\prime}$ is said to be a measure theoretic extension of $T$ if there exist a map $\Theta: X^{\prime} \rightarrow X$ and some $c>0$ such that

$$
\Theta^{-1} \mathcal{B} \subset \mathcal{B}^{\prime}, \Theta \circ T^{\prime}=T \circ \Theta \text { and } \mu^{\prime}\left(\Theta^{-1} A\right)=c \mu(A) \text { for all } A \in \mathcal{B} \text {. }
$$

In this case the map $T$ is said to be a factor of $T^{\prime}$ with factor map $\Theta$.

According to [1, Proposition 3.7.6] and [15], any factor $T$ of a p.d.e. transformation $T^{\prime}$ is also p.d.e. Furthermore, $w_{n}\left(T^{\prime}\right) \propto w_{n}(T)$ and $a_{n}(T) \propto a_{n}\left(T^{\prime}\right)$.

As proved in [1], in some cases an estimate of the wandering rate $w_{n}(T)$ of a p.d.e. transformation gives an immediate estimate of its return sequence $a_{n}(T)$.

Lemma 2.4. [1, Proposition 3.8.7] Let $T$ be a p.d.e. transformation and suppose that $A$ is a Darling-Kac set such that $w_{n}(A) \in \mathcal{R}_{\gamma}$ for some $\gamma \in[0,1]$. Then

$$
a_{n}(T) \propto \frac{n}{w_{n}(A)} \in \mathcal{R}_{1-\gamma} .
$$

The sequence $\left(U_{n}\right)_{n \geq 1}$ on $X$ is said to converge strongly in distribution to a random variable $U$ written as $U_{n} \Longrightarrow \mathfrak{L}(\mu) U$, if $U_{n} \Longrightarrow^{P} U$ for all probability measures $P \ll \mu$.

A DK type theorem for p.d.e. transformations (see also [16] for a different proof of the same statement) reads as follows

Proposition 2.5 (Corollary 3.7.3, [1]). If $T$ is a pointwise dual ergodic transformation of $(X, \mathcal{B}, \mu)$ and the return sequence $a_{n}(T) \in \mathcal{R}_{\gamma}$ for some $\gamma \in[0,1]$ then for every $f \in L^{1}(\mu)$ with $\mu(f)>0$

$$
\frac{1}{a_{n}(T)} \sum_{k=0}^{n-1} f \circ T^{k} \Longrightarrow \stackrel{\mathfrak{L}(\mu)}{ } Y_{\gamma} \int_{X} f d \mu .
$$

where $Y_{\gamma}$ the normalized Mittag-Leffler distribution of order $\gamma$.

\subsection{The Darling-Kac Theorem and tail behavior.}

After establishing the p.d.e. property of the Young tower map $F$ (see Lemma 4.4 below and its proof), we show that one consequence of Theorem 2.1 (together with results recalled in the previous section) is the following version of the DK theorem:

Corollary 2.6. Suppose that $(X, \mathcal{B}, T, m)$ and $\left(\Delta, \mathcal{B}(\Delta), F, m^{\prime}\right)$ satisfy the conditions (2) of Theorem 2.1. Let $\nu$ be the $F$-invariant measure and let $\mu=\pi_{*} \nu$. If $m_{0}(\{R>n\}) \in \mathcal{R}_{-\beta}$ for some $\beta \in[0,1]$, then: 
i1) for all $\hat{f} \in L^{1}(\nu), \nu(\hat{f}) \neq 0$, we have $\frac{S_{n}^{F}(\hat{f})}{a_{n}} \Longrightarrow \mathfrak{L}(\nu) Y_{\beta} \nu(\hat{f})$;

i2) for all $f \in L^{1}(\mu), \mu(f) \neq 0$, we have $\frac{S_{n}^{T}(f)}{a_{n}} \Longrightarrow \stackrel{\mathfrak{L}(\mu)}{ } Y_{\beta} \mu(f)$,

where $a_{n} \in \mathcal{R}_{\beta}$ and $Y_{\beta}$ is a random variable distributed according to the MittagLeffler distribution.

One interesting consequence of Theorem 2.1 concerns the asymptotic tail behavior of the YTs associated with infinite measure preserving transformations that has no analog in the finite measure case. This result, which provides a version of [1, Proposition 5.4.5] formulated in terms of renewal sequences for general Markov towers, states:

Corollary 2.7. Let $T$ be a non-singular, ergodic transformation of $(X, \mathcal{B}, m)$ and assume that $T$ admits an infinite, $\sigma$-finite invariant measure $\mu \ll m$. Suppose that $T$ admits two Young towers $\left(\Delta_{1}, \mathcal{B}\left(\Delta_{1}\right), F_{1}, m_{1}^{\prime}\right)$ with base map $\left(\Delta_{0_{1}}, T^{R_{1}}, m_{0_{1}}\right)$ and $\left(\Delta_{2}, B\left(\Delta_{2}\right), F_{2}, m_{2}^{\prime}\right)$, respectively with base map $\left(\Delta_{0_{2}}, T^{R_{2}}, m_{0_{2}}\right)$.

If the base map of each tower satisfies the conditions (2) of Theorem 2.1 and if $m_{0_{1}}\left(\left\{R_{1}>n\right\}\right) \in \mathcal{R}_{-\beta}$ for some $\beta \in[0,1]$, then $m_{0_{2}}\left(\left\{R_{2}>n\right\}\right) \in \mathcal{R}_{-\beta}$. In particular, if $m_{0_{1}}\left(\left\{R_{1}>n\right\}\right) \propto n^{-\beta}$ for some $\beta \in(0,1]$, then

$$
m_{0_{1}}\left(\left\{R_{1}>n\right\}\right) \propto m_{0_{2}}\left(\left\{R_{2}>n\right\}\right) \propto n^{-\beta} .
$$

Young towers can be found for many $\sigma$-finite measure preserving systems in any dimension, also when no a priori Markov partition is available. Due to the Markov structure of the YT, pointwise dual ergodicity (which is, in general, hard to check) of these systems can be immediately established via [1, Propositions 3.7.5 and 3.7.6] or [2]. Furthermore, as Corollary 2.6 establishes, sufficient conditions for the DK theorem are read off directly from the tail behavior of the return time sequence.

Previously, Zweimüller proved the p.d.e. property for infinite measure preserving piecewise monotone interval maps with indifferent fixed points in [20] that are not Markov. He used a first return map $T_{Y}$ to an interval $Y$ that is bounded away from the neutral fixed points. As $T_{Y}$ has no Markov partition, he built a canonical Markov extension (Hofbauer tower) over $\left(Y, T_{Y}\right)$ to establish and analyze the $T_{Y^{-}}$ invariant absolutely continuous measure. ${ }^{1}$ Alternatively, as observed in [7], wellchosen first return maps within the canonical Markov extension of an interval map $(I, T)$ produce an induced Markov map over $(I, T)$, for which a Young tower can be built. From either approach one can conclude that $(I, T)$ is a pointwise dual ergodic transformation w.r.t. its $\sigma$-finite invariant measure $\mu .^{2}$ In order to establish DarlingKac type theorems further specific information about the the map in question is required. More precisely, one needs to establish the regular variation of the return sequence $a_{n}(T)$, which is a necessary condition (see e.g. [25]). We notice that for the class of maps considered in [20], the regular variation of $a_{n}(T)$ can be verified using particular properties of the original map and not of the extension.

The p.d.e. property in the DK theorem can sometimes be replaced by other easier to check conditions (see $[16,23]$ ) and in this sense considering a special extension that establishes this property becomes needless.

We notice that Zweimüller's version of the DK theorem [23, Theorem 2.1] is more general and covers cases that cannot be covered by earlier the version [16, Theorem

\footnotetext{
${ }^{1}$ Proving finiteness of this measure requires detailed information of the map $T$.

${ }^{2}$ However, there are (logistic) maps for which $\mu(Y)=\infty$ for every nondegenerate interval $Y$, see [4]; hence Darling-Kac sets, if they exist, must be more complicated than intervals in this case.
} 
1]. However, in both versions, the regular variation (with some index $\beta \leq 1$ ) of the wandering rate of a special reference set seems to be essential. This regularly varying condition is not always easy to check. In Section 5 we consider an example based on Example 7.1 in [16], where the regular variation of the wandering rate of the special reference set $Y$ (as defined in [16]) is nontrivial to establish via the methods of $[16,23]$. As we argue in Section 5 , this example can be easily dealt with via Corollary 2.6.

\section{Young towers with non-integrable return time}

Let $\mathbb{N}=\{0,1,2,3,4, \ldots\}$ and $\mathbb{N}^{*}=\{1,2,3,4, \ldots\}$. A transformation $(X, \mathcal{B}, T, m)$ is non-singular w.r.t. $m$ if $T$ is $m$-measurable and $m(A)=0$ implies $m\left(T^{-1} A\right)=0$ for all $A \in \mathcal{B}$.

Suppose $T: X \rightarrow X$ is an ergodic non-singular transformation with respect to a reference measure $m$ ( $m$ is not necessarily invariant). A Young Tower for $(X, \mathcal{B}, T, m)$ is a quartet $\left(\Delta, \mathcal{B}(\Delta), F, m^{\prime}\right)$ with the following properties:

(YT1) There exist a set $\Delta_{0} \subseteq X$ with $0<m\left(\Delta_{0}\right)<\infty$ and a countable partition $\mathcal{P}_{0}:=\left\{\Delta_{0, i}\right\}_{i \in \mathbb{N}}$ of $\Delta_{0}$ with $m\left(\Delta_{0, i}\right)>0$ for each $i$. Let $m_{0}:=\left.m\right|_{\Delta_{0}}$.

(YT2) There is a return time function $R: \Delta_{0} \rightarrow \mathbb{N}^{*}$ which is constant on each $\Delta_{0, i}$, $\left.R\right|_{\Delta_{0, i}}=R_{i}$ and g.c.d. $\left\{R_{i}\right\}=1$. We also assume that $T^{R_{i}}\left(\Delta_{0, i}\right)=\Delta_{0}$ for all $i$.

(YT3) The tower $\Delta$ over $T$ is the set

$$
\Delta:=\left\{(y, l) \in \Delta_{0} \times \mathbb{N}: 0 \leq l<R(y)\right\}
$$

with partition $\mathcal{P}:=\left\{\Delta_{l, i}\right\}$ where $\Delta_{l, i}=\left\{(y, l): y \in \Delta_{0, i}, l<R(y)\right\}$.

(YT4) The dynamics $F: \Delta \circlearrowleft$ on the tower is given by

$$
F(y, l)= \begin{cases}(y, l+1), & \text { if } R(y)>l+1, \\ \left(T^{R(y)}, 0\right), & \text { otherwise. }\end{cases}
$$

The projection

$$
\pi: \Delta \rightarrow X, \quad \pi(y, l)=T^{l} y
$$

defines a semi-conjugacy $T \circ \pi=\pi \circ F$.

(YT5) The measure $m^{\prime}$ on $\Delta$ is obtained by copying $m_{0}$ on each level, i.e., $m^{\prime}:=$ $m_{0} \times d l$ where $d l$ is a counting measure. Notice that $\left.m^{\prime}\right|_{\Delta_{0}}=m_{0}$ and $m^{\prime}\left(\Delta_{l, i}\right)=$ $m_{0}\left(\Delta_{0, i}\right)$.

(YT6) The partition $\mathcal{P}:=\left\{\Delta_{l, i}\right\}$ is a generating $m^{\prime}$-measurable partition. For $x, y \in$ $\Delta_{0}$, let

$$
s(x, y)=\min \left\{n \geq 0:\left(F^{R}\right)^{n}(x),\left(F^{R}\right)^{n}(y) \text { lie in distinct elements of } \mathcal{P}\right\}
$$

be the separation time of $x$ and $y$. There exist constants $C>0$ and $0<\theta<1$ such that for all $x, y \in \Delta_{0, i}$ and all $i$

$$
\left|\log \frac{d m_{0}}{\left.d m\right|_{\Delta_{0, i}} \circ F^{R}}(x)-\log \frac{d m_{0}}{\left.d m\right|_{\Delta_{0, i}} \circ F^{R}}(y)\right| \leq C \theta^{s\left(F^{R}(x), F^{R}(y)\right)} .
$$


This axiomatic structure was introduced by L.-S. Young in $[17,18]$ to study statistical properties of a (probability measure preserving) non-uniformly hyperbolic system, by isolating the uniformly hyperbolic system $F^{R}: \Delta_{0} \rightarrow \Delta_{0}$ and using the structure of the tower and the height function $R$ to make statements about the system $T: X \rightarrow X$.

If the return function $R$ is integrable w.r.t. $m$, then $F$ admits an exact invariant probability measure $\nu$ equivalent to $m^{\prime}$ (see the proof of [18, Theorem 1]). The measure $\mu=\pi_{*} \nu$ is then an invariant probability measure on $(T, X)$ which is absolutely continuous with respect to $m$. Note that $\pi_{*} \nu$ is necessarily a finite a.c.i.m. for $T$. Furthermore, statistical properties of $T$ can be inferred from those of $F$ (see for instance $\S 6$ and $\S 7$ in [18]).

To study infinite measure preserving transformations $(X, T, \mu)$ (in particular, to obtain distributional limit theorems for $T$ ) using properties of the corresponding tower $(\Delta, F, m)$, further clarification about the relationship between the $T$-invariant and $F$-invariant measures is required.

\section{$3.1 \sigma$-finite measures for $\mathbf{F}$}

In this section we formulate a partial version of [18, Theorem 1] for the case of a non-integrable $R$, see $[18, \S 5]$ for a complete version. A non-singular transformation $(X, T, \alpha)$, where $\alpha$ is a generating measurable partition, is said to be Markov if

i) $T$ satisfies the Markov property, i.e., $m(T A \cap B)>0 \Rightarrow B \subseteq T A(\bmod m)$ for all $A, B \in \alpha$, and

ii) $T$ is locally invertible, i.e., for all $A \in \alpha, m(A)>0, T: A \rightarrow T A$ is one-to-one and $T^{-1}: T A \rightarrow A$ is measurable.

A Markov map $(X, \mathcal{B}, m, T, \alpha)$ is aperiodic if for all $A, B \in \alpha$, there exist an $N \in \mathbb{N}$ such that $m\left(A \cap T^{-n} B\right)>0$ for all $n \geq N$.

Proposition 3.1. Let $\left(\Delta, F, m^{\prime}\right)$ be a $Y T$ for some non-singular dynamical system $(X, T, m)$. Let $\mathcal{P}=\left\{\Delta_{l, i}\right\}$ be the partition of $\Delta$ described above. If $\int R d m_{0}=\infty$ then

1. F admits an infinite, but $\sigma$-finite invariant measure $\nu \ll m^{\prime}$ such that $\frac{d \nu}{d m^{\prime}}$ is bounded and bounded away from zero.

2. The system $\left(\Delta, \mathcal{B}(\Delta), F, m^{\prime}, \mathcal{P}\right)$ is aperiodic.

Proof. This proof is based on that of [18, Theorem 1].

i) There is a finite $F^{R}$-invariant measure $\left.\nu_{0} \ll m\right|_{\Delta_{0}}$ on $\Delta_{0}$ with $\frac{d \nu_{0}}{d m_{0}}$ bounded and bounded away from 0 . is obtained, exactly as in the case of integrable $R$, i.e., using an argument based on the Arzela-Ascoli Theorem (see the proof of $[18$, Theorem 1]).

The measure $\nu(A):=\sum_{l=0}^{\infty} \nu_{0}\left(F^{-l} A \cap\{R>l\}\right)$ is $F$-invariant, absolutely continuous with respect to $m^{\prime}$ and $\sigma$-finite invariant. This measure is not finite since $\int R d m_{0}=\infty$ and thus $\nu(\Delta)=\infty$.

ii) It is clear that $\left(\Delta, \mathcal{B}(\Delta), F, m^{\prime}, \mathcal{P}\right)$ is a Markov map. Aperiodicity follows from g.c.d. $\left\{R_{i}\right\}=1$ by the standard argument, which we recall here for completeness. Since $1_{\Delta_{0}}(x) R(x)=1_{\Delta_{0}}(x) \varphi_{\Delta_{0}}(x)$ for all $x$ and g.c.d. $\left\{R_{i}\right\}=1$ we have that g.c.d. $\left\{\varphi_{\Delta_{0}}(x): 0<1_{\Delta_{0}}(x) \varphi_{\Delta_{0}}(x)<\infty\right\}=1$. Thus, for all $[A] \in \mathcal{P}$, there exist an $N \in \mathbb{N}$ such that $[A] \cap F^{-n}\left(\Delta_{0}\right) \neq \emptyset$ for all $n \geq N$. Since we also know that for all $B \in \mathcal{P}$, there exist $N^{\prime} \in \mathbb{N}$ such that $\Delta_{0} \cap F^{-N^{\prime}}([B]) \neq \emptyset$, the aperiodicity follows. 
If in the setting of this proposition, $\int R d m_{0}<\infty$, then the resulting $F$-invariant measure $\nu \ll m^{\prime}$ is finite, and $\mu=\left(\int R \frac{d \nu}{d m^{\prime}} m_{0}\right)^{-1} \pi_{*} \nu$ is an absolutely continuous $T$-invariant probability measure.

If $\int R d m_{0}=\infty$, then since $\nu$ is always $\sigma$-finite, the measure $\pi_{*} \nu$ is still an absolutely continuous $T$-invariant measure for $T$. This is an immediate consequence of the fact that $\nu \ll m$ is invariant for F; see also [21, Proposition 1.1] for the relationship between the invariant measures of a general induced transformation $T^{R}$ and that of the original system. However, it is not always true that $\pi_{*} \nu$ is a $\sigma$-finite measure for $T$.

Further conditions are required for a canonical link between the $T$-invariant measure and the projection of $F$-invariant measures.

\section{$3.2 \sigma$-finite measures for $T$}

In this section we give conditions under which the $\sigma$-finite measure $\nu$ on the YT projects to a $\sigma$-finite measure $\mu=\pi_{*} \nu$ on $(X, T)$. We start with an example showing that this is not automatic: the YT construction can produce an infinite $T$-invariant measure which is not $\sigma$-finite, if a non-integrable return time function is suitably chosen (see also a similar Example 2.2 in [21]).

Example 1. Let $T:[0,1] \rightarrow[0,1]$ be the doubling map $T(x)=2 x \bmod 1$. The countable partition $\left\{I_{n}\right\}$, where $I_{n}=\left(\frac{1}{2^{n+1}}, \frac{1}{2^{n}}\right]$ is Markov for $T$. Subdivide each interval $I_{n}$ into $2^{2^{n}}$ intervals of equal length and call them $I_{n, j}$. It follows that $m\left(I_{n, j}\right)=1 / 2^{n+2^{n}}$ and $\sum_{j} m\left(I_{n, j}\right)=m\left(I_{n}\right)=1 / 2^{n}$.

Let $\Delta_{0}=(0,1]$ and consider the countable partition $\left\{\Delta_{0, i}\right\}:=\left\{I_{n, j}\right\}$. Define $R: \Delta \rightarrow \mathbb{N}$ such that $\left.R\right|_{I_{n, j}}=n+2^{n}$. This choice gives $T^{R}\left(I_{n, j}\right)=\Delta_{0}$ and thus $(Y T 1)$ and $(Y T 2)$ are verified. Notice that $(Y T 6)$ is trivially satisfied for $m_{0}:=m$ because $T$ is expanding and linear on each branch. Furthermore, it is obvious that the $T^{R}$-invariant measure is exactly $m$. Thus one obtains the exact form of the tower $F$ by applying $(Y T 3),(Y T 4)$ and $(Y T 5)$. Also, since $\sum_{j} m\left(I_{n, j}\right)=1 / 2^{n}$, one has

$$
\int R d m=\left.\sum_{n} \sum_{j} R\right|_{I_{n, j}} m\left(I_{n, j}\right)=\sum_{n}\left(n+2^{n}\right) \sum_{j} m\left(I_{n, j}\right)=\sum_{n} \frac{n+2^{n}}{2^{n}}=\infty .
$$

So, we are in the non-integrable case. By Proposition 3.1 we know that $F$ admits an infinite, but $\sigma$-finite invariant measure $\nu \ll m$ given by $\nu(A):=\sum_{l=0}^{\infty} m\left(F^{-l} A \cap\right.$ $\{R>l\})$.

Since $F^{-l} \circ \pi^{-1}=\pi^{-1} \circ T_{1}^{-l}$, by projecting back with $\pi$ we have

$$
\begin{aligned}
\pi_{*} \nu(E) & =\nu\left(\pi^{-1} E\right)=\sum_{l \geq 0} m\left(\{R>l\} \cap F^{-l} \pi^{-1} E\right) \\
& =\sum_{l \geq 0} m\left(\pi^{-1}(\{R>l\}) \cap T^{-l} E\right) \\
& =\sum_{l \geq 0} m\left(\{R>l\} \cap T^{-l} E\right)
\end{aligned}
$$

and thus $\pi_{*} \nu([0,1])=\infty$. It is always the case that the measure $\mu:=\pi_{*} \nu$ is invariant for $T$ and $\mu \ll m$. However, in this particular case the measure $\mu$ cannot be $\sigma$-finite since already Lebesgue measure $m$ is ergodic and $T$-invariant, and every pair of equivalent $\sigma$-finite invariant ergodic measures differ by a finite multiplicative constant.

The example given above shows that when the return function is non-integrable, the 
axiomatic structure of the tower is not enough to guarantee that the measure $\pi_{*} \nu$ is $\sigma$-finite and thus if one wants to use YT constructions to study infinite measure preserving transformations, then some further constraints are required.

If $R$ is the first return time of $T$ to $\Delta_{0}$, i.e., $T^{R}=T^{\varphi_{\Delta_{0}}}$, then the measure $\pi_{*} \nu$ is always $\sigma$-finite as a consequence of [14], see also [1, 20,21]. Indeed, the explicit formula

$$
\mu(E):=\sum_{n \geq 0} \nu_{0}\left(T^{-n} E \cap\left\{\varphi_{\Delta_{0}}>n\right\}\right)
$$

for $\nu_{0}=\left.\nu\right|_{\Delta_{0}}$ shows that the sets $X \backslash \cup_{k} T^{-k}\left(\Delta_{0}\right)$ and $\left.T^{n}\left(\left\{\phi_{\Delta_{0}}\right)>n\right\}\right), n \geq 0$, form a countable partition of $X$ into sets of finite measure. Conversely, if $\mu \ll m$ is $T$-invariant then $\nu_{0}:=\left.\mu\right|_{\Delta_{0}}$ is $T_{\Delta_{0}}$-invariant.

A standard example of an infinite m.p.t. that can be modeled by a YT by taking $R$ as the first return time of $T$ to $\Delta_{0}$ is the PM map (with $\alpha \geq 1$ ) mentioned in the introduction, the construction being identical to the finite case (given by $\alpha<1$ ).

Another well-understood non-integrable $R$ case is given by the class of transformations $(T, X, m)$ for which the base tower map $\left(T^{R}, \Delta_{0}, m_{0}\right)$ can be obtained by letting $R$ be the first passage time of $T$ to some set $A$ with $T(A)=\Delta_{0}$, i.e., $\tau(x):=1+\min \left\{n \geq 0: T^{n}(x) \in A\right\}$. As proved by Schweiger (see e.g. $[14,21,1]$ ) the map $T^{\tau}$ is similar to $T^{\varphi \Delta_{0}}$ (that is, they have a common measure theoretic extension), which implies that if $T^{\tau}$ admits a probability invariant measure $\nu_{0} \ll m_{0}$ then $T^{\varphi_{\Delta_{0}}}$ admits a probability invariant measure $\tilde{\nu_{0}} \ll m_{0}$ and there exist $\Theta: \Delta_{0} \rightarrow X$ and $c>0$ such that

$$
\Theta^{-1}\left(\Delta_{0} \cap \mathcal{B}(X)\right) \subset \mathcal{B}(X), \Theta \circ T^{\varphi \Delta_{0}}=T^{\tau} \circ \Theta \text { and } \tilde{\nu_{0}}\left(\Theta^{-1} A\right)=c \nu_{0}(A)
$$

for all $A \in \mathcal{B}(X)$. As a consequence,

$$
\begin{aligned}
\pi_{*} \nu(E) & =\nu\left(\pi^{-1} E\right)=\sum_{l \geq 0} \nu_{0}\left(\{\tau>l\} \cap F^{-l} \pi^{-1} E\right) \\
& =\sum_{l \geq 0} \nu_{0}\left(\pi^{-1}\left(\{\tau>l\} \cap\left(\tilde{T}^{\tau}\right)^{-l} E\right)\right. \\
& =\sum_{l \geq 0} \nu_{0}\left(\{\tau>l\} \cap\left(\tilde{T}^{\tau}\right)^{-l} E\right) .
\end{aligned}
$$

Therefore, the last equation is exactly the $\sigma$-finite measure of (5) and thus, $\pi_{*} \nu$ is $\sigma$-finite.

Example 2. We consider the Farey map given by $T:[0,1] \rightarrow[0,1]$ with

$$
T(x):= \begin{cases}T_{1}(x)=\frac{x}{1-x}, & \text { if } x<1 / 2 \\ T_{2}(x)=\frac{1-x}{x}, & \text { if } x \geq 1 / 2\end{cases}
$$

Let $m$ denote Lebesgue measure. There exists a countable Markov partition $\alpha=$ $\left\{A_{n}\right\}_{n \geq 0}$, where $A_{n}=\left(\frac{1}{n+1}, \frac{1}{n}\right]$. Take $A=A_{1}=\left(\frac{1}{2}, 1\right]$ and $\Delta_{0}=T(A)$. Then inducing on $A$ w.r.t. first passage time $\tau$ yields the Gauss map $G(x)=\frac{1}{x}-\left\lfloor\frac{1}{x}\right\rfloor$, see e.g. [11], for which good distortion properties are well known. From here on, the tower construction is standard. Take $\Delta_{0}:=(0,1]$ and let $R(x):=\tau(x)$ for all $x \in \Delta_{0}$. Let $\left\{\Delta_{0, i}\right\}:=\left\{A_{0}\right\}_{n \geq 0}$. It is easy to see that (YT1) and (YT2) hold. We can use standard arguments (see e.g. [11]) to conclude that for all $x, y \in \Delta_{0, i}$ and for all $i$

$$
\left|\log \frac{\left(T^{\tau}\right)^{\prime}(x)}{\left(T^{\tau}\right)^{\prime}(y)}\right|=\left|\log \frac{G^{\prime}(x)}{G^{\prime}(y)}\right| \leq \frac{C}{D} \theta^{s(x, y)}
$$


where $\theta=1 / 2$ and $s(x, y)$ is the separation time w.r.t. $T^{\tau}$. Thus, taking $m_{0}:=m$, (YT6) is satisfied. The tower construction is completed by applying (YT3), (YT4) and (YT5), which give the exact form of $F$. Since $\tau$ is the first passage time, (7) guarantees that $\pi_{*} \nu$ is $\sigma$-finite.

Necessary and sufficient conditions for when a $T^{R}$-invariant measure corresponds to a $\sigma$-finite $T$-invariant measure (via formula (8) below) in the case of a general induced map $T^{R}$ with non-integrable $R$ can be obtained based on results of Zweimüller, [21]. For clarity of exposition, we provide these results below. To avoid confusion later on, when we apply these results to the context of YTs, we will state them keeping our notation $T^{R}: \Delta_{0} \rightarrow \Delta_{0}$ even though in [21], $R$ and $\Delta_{0}$ do not need to be chosen so that they produce a $\mathrm{YT}\left(\Delta, \mathcal{B}(\Delta), F, m^{\prime}\right)$ for the original transformation $(X, \mathcal{B}, T, m)$. Namely, [21] works with the following general setting:

The setting of [21]. Let $(X, \mathcal{B}, T, m)$ be a nonsingular transformation. For an arbitrary set $\Delta_{0} \subseteq X, m\left(\Delta_{0}\right)>0$, the measurable function $R: \Delta_{0} \rightarrow \mathbb{N}^{*}$ is a general inducing time $(\bmod m)$ for $T$ on $\Delta_{0}$, if it is finite $m$-a.e. and $T^{R} x:=T^{R(x)} x \in \Delta_{0}$ for $m$-a.e. $x \in \Delta_{0}$. Hence $T^{R}$ is a nonsingular transformation of $\left(\Delta_{0}, \mathcal{B}\left(\Delta_{0}\right), m_{0}\right)$, where $m_{0}:=\left.m\right|_{\Delta_{0}}$ and $\mathcal{B}\left(\Delta_{0}\right)=\left\{A \in \mathcal{B}: A \subset \Delta_{0}\right\}$. (This is now more general than the YT setting because we do not assume that $T^{R}\left(\Delta_{i}\right)=\Delta_{0}$ or the distortion constraint of (YT6).) Given any measure $\tilde{\nu}$ on $\Delta_{0}$, a new $T$-invariant measure on $(X, \mathcal{B})$ can be defined as follows:

$$
R \times_{T} \tilde{\nu}(A):=\sum_{l \geq 0} \tilde{\nu}\left(\{R>l\} \cap T^{-l} A\right) .
$$

The work of [21] provides an answer for the following two questions:

(i) The original liftability problem, i.e., given that $\mu$ is a $\sigma$-finite invariant measure for $T$, is there a $T^{R}$-invariant measure $\tilde{\nu} \ll \mu$ such that $\mu=R \times_{T} \tilde{\nu}$ ?

(ii) The inverse liftability problem, i.e., given that $\tilde{\nu} \ll m_{0}$ is $T^{R}$-invariant, is the measure $R \times_{T} \tilde{\nu}$ a $\sigma$-finite invariant measure for $T$ ?

We will consider Zweimüller's results on the inverse liftability problem in the context of YTs. We recall the following:

Lemma 3.2. [21, Lemma 4.1] Let $(X, \mathcal{B}, T, m)$ be a nonsingular transformation. Let $E \subseteq \Delta_{0} \subseteq X, m(E)>0$. Let $\rho$ be an inducing time for $T$ on $E$, let $R$ be an inducing time for $T$ on $\Delta_{0}$ and let $\psi$ be an inducing time for $T^{R}$ on $E$ such that $\rho=R_{\psi}:=\sum_{k=0}^{\psi-1} R \circ\left(T^{R}\right)^{k}$. This implies that $T^{\rho}=\left(T^{R}\right)^{\psi}$. Moreover, let $\tilde{\nu} \ll m$ be a measure (not necessarily $T^{R}$-invariant) on $\left(\Delta_{0}, \mathcal{B}\left(\Delta_{0}\right)\right)$. Then

$$
\rho \times_{T} \tilde{\nu}=R \times_{T}\left(\psi \times_{T^{R}} \tilde{\nu}\right) .
$$

Fact 3.3. As observed in [21], the first return map of $T^{R}$ to some set $E \in \mathcal{B}\left(\Delta_{0}\right)$, $m(E)>0$, can be represented as $T^{\rho}=\left(T^{R}\right)_{E}=\left(T_{E}\right)^{\tau}$, where $\rho: E \rightarrow \mathbb{N}$ and $\tau: E \rightarrow \mathbb{N}$ are general inducing times for $T$ and $T_{E}$, respectively. We notice that $\left(T^{R}\right)_{E}=\left(T^{R}\right)^{\varphi_{E}^{R}}$, where $\varphi_{E}^{R}(x):=\min \left\{n \geq 1:\left(T^{R}\right)^{n}(x) \in E\right\}$. Also, the inducing time $\rho$ can be equivalently represented as

$$
\rho=\sum_{k=0}^{\varphi_{E}^{R}-1} R \circ\left(T^{R}\right)^{k} \text { and } \rho=\varphi_{E, \tau}:=\sum_{k=0}^{\tau-1} \varphi_{E} \circ\left(T_{E}\right)^{k} .
$$


In the context of YTs we have briefly mentioned that the integrability of the return time is a sufficient condition for the inverse liftability problem. The result below says that this condition is also sufficient for the original liftability problem and it is already well known.

Lemma 3.4. ([21, Theorem 1.1]) Let $(X, \mathcal{B}, T, \mu)$ be an e.m.p.t. and let $\tau$ be a general inducing time for $T$ on $E \in \mathcal{B}, \mu(E)>0$. If $\int_{E} \tau d \mu<\infty$, then $T^{\tau}$ has an invariant measure $\nu$ satisfying $\mu=\tau \times_{T} \nu$.

The next two lemmas by Zweimüller lead up to the main result of this section.

Lemma 3.5. ([21, Proposition 4.1]) Let $T$ be c.e.m.p.t. of the $\sigma$-finite measure space $(X, \mathcal{B}, T, \mu)$. Let $R$ be an inducing time for $T$ on $\Delta_{0} \subseteq X, 0<\mu\left(\Delta_{0}\right)<\infty$. Suppose that $E \in\left(\Delta_{0}, \mathcal{B}\left(\Delta_{0}\right)\right), \mu(E)>0$ with $\left(T^{R}\right)_{E}=\left(T_{E}\right)^{\tau}$. Then

$$
\tilde{\nu} \text { satisfies } \mu=R \times_{T} \tilde{\nu}
$$

if and only if

$$
\hat{\nu} \text { satisfies }\left.\mu\right|_{E}=\tau \times_{T_{E}} \hat{\nu} .
$$

If one of the two measures $\tilde{\nu}$ and $\hat{\nu}$ exists (and thus both) then $\hat{\nu}=\left.\tilde{\nu}\right|_{E}$ or equivalently $\tilde{\nu}=\left(\varphi_{E}^{R}\right) \times_{T_{R}} \hat{\nu}$.

Lemma 3.6. [22, Proposition 1] Let $T$ be a measurable transformation $(X, \mathcal{B})$ and let $E \in \mathcal{B}$. Let $\rho$ and $\tau$ be inducing times for $T$ and $T_{E}$ on $E$ such that $T^{\rho}=\left(T_{E}\right)^{\tau}$. Moreover, let $\tilde{\nu}$ be a measure on $E$. Then

$$
\rho \times_{T} \tilde{\nu}(E)=\int_{E} \tau d \tilde{\nu}
$$

Equipped with the above, we can proceed to the

Proof of Theorem 2.1. First, we observe that by Proposition 3.1 the F-invariant measure $\nu \ll m^{\prime}$ is $\sigma$-finite. Then, under the assumptions of the proposition, equation (4) holds and thus, $\pi_{*} \nu=R \times_{T} \nu_{0}$. By the same assumptions, $\left(T^{R}, \Delta_{0}, \nu_{0}\right)$ is an ergodic transformation preserving the probability measure $\nu_{0} \ll m$.

Let $E \subseteq \Delta_{0}$ and consider the first return time of $T^{R}$ to $E$. By Fact 3.3 we may write $T^{\rho}=\left(T^{R}\right)_{E}=\left(T_{E}\right)^{\tau}$ for some measurable functions $\rho: E \rightarrow \mathbb{N}$ and $\tau: E \rightarrow \mathbb{N}$. Let $\tilde{\nu}_{E}=\left.\nu_{0}\right|_{E}$ be a finite measure on $E$.

By Lemma 3.2 applied to $T^{\rho}=\left(T^{R}\right)_{E}$ we have

$$
\rho \times_{T} \tilde{\nu}_{E}(A)=R \times_{T}\left(\varphi_{E}^{R} \times_{T^{R}} \tilde{\nu}_{E}\right)(A) .
$$

On the other hand, another application of Lemma 3.2 to $T^{\rho}=\left(T_{E}\right)^{\tau}$ gives

$$
\rho \times_{T} \tilde{\nu}_{E}(A)=\varphi_{E} \times_{T}\left(\tau \times_{T_{E}} \tilde{\nu}_{E}\right)(A) .
$$

Since $\mu \ll m$ (by assumption) and $\nu_{0} \ll m$ (whence $\tilde{\nu}_{E} \ll m$ ), Lemma 3.5 implies that

$$
\nu_{0} \text { is a solution for } \mu=R \times_{T} \nu_{0}
$$

if and only if

$$
\tilde{\nu}_{E} \text { is a solution for }\left.\mu\right|_{E}=\tau \times_{T_{E}} \tilde{\nu}_{E} .
$$

In this case, $\nu_{0}=\left(\varphi_{E}^{R}\right) \times_{T_{R}} \tilde{\nu_{E}}$, which further implies that 


$$
\mu=R \times_{T} \nu_{0}=R \times_{T}\left(\varphi_{E}^{R} \times_{T^{R}} \tilde{\nu}_{E}\right) .
$$

Therefore, if $\tilde{\nu}_{E}$ is a solution for $\left.\mu\right|_{E}=\tau \times_{T_{E}} \tilde{\nu}_{E}$ (or equivalently if $\nu_{0}$ is a solution for $\mu=R \times_{T} \nu_{0}$ ), then (9), (10) and (11) imply that

$$
\mu=R \times_{T} \nu_{0}=R \times_{T}\left(\varphi_{E}^{R} \times_{T}^{R} \tilde{\nu_{E}}\right)=\rho \times_{T} \tilde{\nu}_{E}=\varphi_{E} \times_{T}\left(\tau \times_{T_{E}} \tilde{\nu}_{E}\right)
$$

We now take a look at the finite e.m.p.t. $\left(T_{E}, E,\left.\mu\right|_{E}\right.$ ) (the ergodicity of $T_{E}$ follows from our assumption that $T$ is ergodic). Recall that $\tau$ is an inducing time for $T_{E}$ on $E$. Therefore, if $\int_{E} \tau d m<\infty$, then by Lemma 3.4, $\tilde{\nu}_{E}$ satisfies $\left.\mu\right|_{E}=\tau \times_{T_{E}} \tilde{\nu}_{E}$ and $\left(T_{E}\right)^{\tau}$ has a (unique) finite invariant measure $\tilde{\nu}_{E} \ll m$. By our discussion above, this further implies that $\nu_{0}$ satisfies $\mu=R \times_{T} \nu_{0}$, which proves the "if" part of the proposition.

Conversely, assume that there exist $E$ and $\tau$ as above such that $\int_{E} \tau d m=\infty$. By Lemma 3.6 we have

$$
\rho \times_{T} \tilde{\nu}_{E}(E)=\int_{E} \tau d \tilde{\nu}_{E}=\int_{E} \tau d m=\infty .
$$

Suppose that $\tilde{\nu}_{E}$ is a solution for $\left.\mu\right|_{E}=\tau \times_{T_{E}} \tilde{\nu}_{E}$. But then the above equation together with (12) implies

$$
\pi_{*} \nu(E)=R \times_{T} \nu_{0}=\int_{E} \tau d m=\infty
$$

which contradicts the hypothesis and we are done.

Remark 3.7. As $\mu$ is $\sigma$-finite, there is $E \in \mathcal{B}$ such that $0<\mu(E)<\infty$ and by the proof of the proposition above, we know $0<\pi_{*} \nu(E)<\infty$. Thus the above proposition implies that if $\mu=\pi_{*} \nu$ then there exists a set $E \subseteq \Delta_{0}, m(E)>0$ with $\tau: E \rightarrow \mathbb{N}$ given by $\left(T^{R}\right)_{E}=\left(T_{E}\right)^{\tau}$ such that $\int_{E} \tau d m<\infty$. This would become a sufficient condition as well if we also assume that the inducing time $\rho$ is defined and finite $\pi_{*} \nu$-a.e., since this would guarantee that $\bigcup_{n \geq 0} T^{-n} E=X \bmod \pi_{*} \nu$ and thus that $\pi_{*} \nu$ is $\sigma$-finite.

\section{Pointwise dual ergodicity}

To prove Corollary 2.6 we only need to establish the p.d.e. property for the tower map $F$. Then the result follows immediately from Theorem 2.1, an estimate of the wandering rate $w_{n}(F)$ together with Proposition 3.7.5 in [1] and Proposition 2.5. Corollary 2.7 follows by a similar argument together with the following standard results on regularly varying functions (sequences).

Proposition 4.1 (Karamata's Theorem [5]). The function $a(t)$ is slowly varying and locally bounded if and only if for any constant $c$ :

- $\int_{c}^{x} t^{\gamma} a(t) d t \sim \frac{x^{\gamma+1}}{\gamma+1} a(x)$, if $\gamma>-1$

- $\int_{x}^{\infty} t^{\gamma} a(t) d t \sim \frac{x^{\gamma+1}}{|\gamma+1|} a(x)$, if $\gamma<-1$

Furthermore, the following theorem gives an exact characterization of functions (sequences) that produce regularly varying functions (sequences): 
Proposition 4.2 (Monotone Density Theorem [5]). Let $U(x)=\int_{0}^{x} u(y) d y$ and suppose $U(x) \sim x^{\gamma} a(x)$ for some $\gamma \in \mathbb{R}$ and function $a \in \mathcal{R}_{0}$. If $u$ is monotone, then

$$
u(x) \sim \gamma x^{\gamma-1} a(x) .
$$

\subsection{Pointwise dual ergodicity for $F$}

In the following we show that a YT is p.d.e. under a less restrictive condition than (YT2) formulated in Section 3. That is, we replace the previous YT2 with:

(YT2') There is a return time function $R: \Delta_{0} \rightarrow \mathbb{N}^{*}$ which is constant on each $\Delta_{0, i}$. We also assume that $T^{R_{i}}\left(\Delta_{0, i}\right)$ is a union of $\Delta_{0, k}$ 's and $\inf _{i \in \mathbb{N}} m_{0}\left(T^{R_{i}}\left(\Delta_{0, i}\right)\right)>0$.

In order to obtain good properties of the $F$-invariant measure, under the weaker (YT2') above, we need the following extra-assumption, (see also [8] for obtaining estimates of the correlation decay on towers via cone techniques under (YT2')):

(YT7) $\left(\Delta, \mathcal{B}(\Delta), F, m^{\prime}\right)$ is aperiodic.

We first recall the following concepts and results. Let $(X, \mathcal{B}, m, T)$ be a Markov map with Markov partition $\alpha$ and for $a_{0}, \ldots, a_{n-1} \in \alpha$ let $\left[a_{0}, \ldots, a_{n-1}\right]=\cap_{i=0}^{n-1} T^{-i} a_{i}$ denote an $n$-cylinder. Let $\alpha_{0}^{n-1}=\bigvee_{k=0}^{n-1} T^{-k}(\alpha)$ and $\alpha_{+}=\left\{a \in \cup_{n \in \mathbb{N}^{*}} \alpha_{0}^{n-1}: m(a)>\right.$ $0\}$. A collection of cylinders $\zeta \subseteq \alpha_{+}$is said to be a Schweiger collection for $T$ if

i) for every $b \in \zeta$ and $a \in \alpha_{+}$if the concatenation $[a, b] \neq \emptyset$ then $[a, b] \in \zeta$;

ii) $\cup_{b \in \zeta} b=X(\bmod m)$;

iii) if there exist $r \in(0,1)$ and $C>1$ such that for every $n$-cylinder $b \in \zeta$ and $m \times m$-a.e. $(x, y) \in b \times b$

$$
\left|\log \frac{d m}{d m \circ T^{n}}\right|_{b}(x)-\left.\log \frac{d m}{d m \circ T^{n}}\right|_{b}(y) \mid \leq C r^{s(x, y)}
$$

where

$$
s(x, y):=\min \{n \geq 1: x, y \text { lie in distinct cylinders of } \zeta\} .
$$

The existence of a Schweiger collection for a c.m.p.t. and aperiodicity have the following consequences:

Lemma 4.3 (Theorem 3.1 and Theorem 3.2, [2]). Let $(X, \mathcal{B}, m, T, \alpha)$ be a conservative, aperiodic Markov map and suppose that $\zeta \subseteq \alpha_{+}$is a Schweiger collection for $T$. Then $T$ admits a $\sigma$-finite invariant measure $\mu \sim m$ such that

$$
\log \frac{d \mu}{d m} \in L^{\infty}(b) \text { for all } b \in \zeta
$$

and $\mu$ is exact under T. Moreover, any $A \in \alpha_{+}$is a Darling Kac set whose return time process is continued fraction mixing.

We now show

Lemma 4.4. Let $\left(\Delta, \mathcal{B}(\Delta), F, m^{\prime}\right)$ be a YT that satisfies (YT2') and (YT7) above. ${ }^{3}$ Then the following hold:

1. F admits an infinite, but $\sigma$-finite invariant measure $\nu$ equivalent to $m^{\prime}$ such that $\frac{d \nu}{d m^{\prime}}$ is bounded and bounded away from zero. Furthermore, $\nu$ is exact under $F$.

\footnotetext{
${ }^{3}$ so the original condition (YT2) of Section 3 need not be satisfied
} 
2. For any $n \in \mathbb{N}$, every $n$-cylinder is a Darling-Kac set whose return time process is continued fraction mixing.

3. $F$ is pointwise dual ergodic w.r.t. $\nu$.

Proof. Let $\mathcal{P}$ be the generating partition of $\Delta$ and let $\left[A_{0}, \ldots, A_{n-1}\right]=\bigcap_{i=0}^{n-1} F^{-i} A_{i}$ for $A_{0}, \ldots, A_{n-1} \in \mathcal{P}$ denote an $n$-cylinder. It is clear that $\left(\Delta, \mathcal{B}(\Delta), F, m^{\prime}, \mathcal{P}\right)$ is a conservative Markov map. By (YT1), $m\left(\Delta_{0, i}\right)>0$ for each $i$ and hence $m(A)>0$ for all $A \in \mathcal{P}$ and and thus $m^{\prime}$ is positive on all $n$-cylinders. Also, it is clear that $\left(\Delta_{0}, \mathcal{B}(\Delta), F^{R}, m_{0}\right)$ is a conservative Markov map w.r.t. the countable partition $\mathcal{P}_{0}=\left\{\Delta_{0, i}\right\}$; let $\mathcal{R}:=\left\{T^{R}\left(\Delta_{0, i}\right)\right\}$ be the image partition and $\tilde{\mathcal{R}}_{+}:=\left\{a \in \cup_{k \in \mathbb{N}} \bigvee_{0}^{k-1}\left(F^{R}\right)^{-k} \mathcal{R}\right\}$.

It follows from (YT6) that there exist $C>1$ and $0<\theta<1$ such that for all $x, y \in b$, for all $b \in \mathcal{R}_{+}$, and for all $k \in \mathbb{N}$ and $k$-cylinders $b$

$$
\left|\sum_{j=0}^{k-1} \log \frac{d m_{0}}{d m_{b} \circ\left(F^{R}\right)^{j}}(x)-\sum_{j=0}^{k-1} \log \frac{d m_{0}}{d m_{b} \circ\left(F^{R}\right)^{j}}(y)\right| \leq C \theta^{s(x, y)}
$$

and thus $\mathcal{R}_{+}$is a Schweiger collection for $F^{R}$ w.r.t. the measure $m_{0}$. By [2, Lemma 2.1] (which requires condition (YT2')), there exist an $F^{R}$-invariant probability measure $\nu_{0} \ll m_{0}$ such that $\frac{d \nu_{0}}{d m_{0}}$ is bounded and bounded away from zero.

By aperiodicity and (YT2'), there exist $N \in \mathbb{N}$ and $b \in \mathcal{P}_{0}$ such that $\left(F^{R}\right)^{N}(b)=$ $\Delta_{0}$. Therefore for all $a \in \mathcal{P}_{0}$ and $x \in a$ we can find $x^{\prime} \in b$ such that $\left(F^{R}\right)^{N}\left(x^{\prime}\right)=x$, and we have

$$
\frac{d \nu_{0}}{d m_{0}}(x) \geq \frac{d m_{0}}{d m_{b} \circ\left(F^{R}\right)^{N}} \cdot \frac{\nu_{0}}{m_{0}}\left(x^{\prime}\right)>0
$$

uniformly over all $a \in \mathcal{P}$ and $x \in a$. This also implies that $\nu_{0} \sim m_{0}$.

Fix some arbitrary $\Delta_{l, i}$ and let

$$
\mathcal{P}_{\Delta_{l, i}}=\left\{\left[A_{0}, \ldots, A_{n-1}\right]: A_{0}, \ldots, A_{n-1} \in \mathcal{P}, n \in \mathbb{N}^{*}, A_{n-1}=\Delta_{l, i}\right\}
$$

be the collection of cylinders that land on $\Delta_{l, i}$ after some number of iterates. Notice that $\mathcal{P}_{\Delta_{l, i}} \subset \mathcal{P}_{+}:=\left\{A \in \cup_{k \in \mathbb{N}} \bigvee_{0}^{k-1} F^{-k} \mathcal{P}\right\}$.

For all $x^{\prime}, y^{\prime} \in \Delta_{l, i}$ there exist unique $x, y \in \Delta_{0, i}$ such that $F^{l}\left(x^{\prime}\right)=x, F^{l}\left(y^{\prime}\right)=y$; let us extend the definition of separation time to $\Delta_{l, i}$ by setting $s(x, y)=s\left(x^{\prime}, y^{\prime}\right)$. It follows from (14) that there exist $C>1$ and $0<\theta<1$ such that for all $x^{\prime}, y^{\prime} \in \Delta_{l, i}$ and for $t=R-l$

$$
\left|\sum_{k=0}^{t-1} \log \frac{d m^{\prime}}{\left.d m^{\prime}\right|_{\Delta_{l, i}} \circ F^{k}}\left(x^{\prime}\right)-\sum_{k=0}^{t-1} \log \frac{d m^{\prime}}{\left.d m^{\prime}\right|_{\Delta_{l, i}} \circ F^{k}}\left(y^{\prime}\right)\right| \leq C \theta^{s\left(x^{\prime}, y^{\prime}\right)}
$$

which further implies for all $B \in \mathcal{P}_{\Delta_{l, i}}$ and for all $x, y \in B$ we have

$$
\left|\sum_{k=0}^{t-1} \log \frac{d m^{\prime}}{\left.d m^{\prime}\right|_{B} \circ F^{k}}(x)-\sum_{k=0}^{t-1} \log \frac{d m^{\prime}}{\left.d m^{\prime}\right|_{B} \circ F^{k}}(y)\right| \leq C \theta^{s(x, y)} .
$$

Also, for every $B \in \mathcal{P}_{\Delta_{l, i}}$ and $A \in \mathcal{P}_{+}$if $[A, B] \neq \emptyset$ then $[A, B] \in \mathcal{P}_{\Delta_{l, i}}$. Furthermore, by aperiodicity for all $A \in \mathcal{P}$ there exist $N=N(A) \in \mathbb{N}$ such that for all $j \geq N$, $m^{\prime}\left(F^{-j} A \cap\left(\cup_{B \in \mathcal{P}_{\Delta_{l, i}}} B\right)\right)>0$. As a consequence, $\cup_{B \in \mathcal{P}_{\Delta_{l, i}}} B=\Delta\left(\bmod m^{\prime}\right)$ and thus, $\mathcal{P}_{\Delta_{l, i}}$ is a Schweiger collection for $F$ w.r.t. $m^{\prime}$. Therefore, by Lemma $4.3, F$ admits an exact, $\sigma$-finite invariant measure $\nu \sim m^{\prime}$ and $\frac{d \nu}{d m^{\prime}}$ is bounded away from 0 and $\infty$ uniformly on $\Delta_{l, i}$. 
The same lemma implies that each element of $\mathcal{P}_{\Delta_{l, i}}$ is a Darling-Kac set for $F$ w.r.t. $\nu$. Thus, by Theorem 3.8.3 in [1], $F$ is pointwise dual ergodic w.r.t. $\nu$. That is, there exist a positive sequence $\left\{a_{n}(F)\right\}_{n \geq 1}$ such that for all $f \in L^{1}(\nu)$ with $\int_{X} f d \nu>0$

$$
\frac{1}{a_{n}(F)} \sum_{k=0}^{n-1} \hat{\mathbf{F}}^{k}(f) \rightarrow \int_{\Delta} f d \nu, \nu-\text { a.e. as } n \rightarrow \infty
$$

where $\hat{\mathbf{F}}: L^{1}(\nu) \rightarrow L^{1}(\nu)$ is the dual operator of $F$.

Since $\nu \sim m^{\prime}$ is exact, it follows that this measure is unique and thus $\nu$ is independent of $\Delta_{l, i}$. In fact $\left.\nu\right|_{\Delta_{0}}=\nu_{0}$ and we saw already that $\frac{d \nu_{0}}{d m_{0}}$ is bounded away from zero and $\infty$, so $\frac{d \nu}{d m^{\prime}}$ is bounded away from zero and $\infty$ uniformly on $\Delta$. Similarly, all elements of $\cup_{l, i} \mathcal{P}_{\Delta_{l, i}}$ are Darling-Kac sets for $F$ w.r.t. $\mu$. This concludes the proof.

\subsection{Pointwise dual ergodicity for $T$.}

Pointwise dual ergodicity for $T$ can be immediately derived from that of $F$ :

Lemma 4.5. Suppose that $(X, \mathcal{B}, T, m)$ and $\left(\Delta, \mathcal{B}(\Delta), F, m^{\prime}\right)$ satisfy the conditions of Theorem 2.1. Then $T$ is pointwise dual ergodic w.r.t. its invariant measure $\mu=$ $\pi_{*} \nu$ and the return sequence $a_{n}(F)$ from (15) coincides, up to asymptotic equivalence, with the return sequence for $T$.

Proof. We first observe that Theorem 2.1 ensures that $F$ is indeed a measure theoretic extension of $T$, or equivalently $T$ is a factor of $F$. By Proposition 3.7.6 in [1], we know that any factor of a p.d.e. transformation is also p.d.e. This together with (3) of Lemma 4.4 implies that $T$ is indeed pointwise dual ergodic. Furthermore, according to Proposition 3.7.6 in [1], $a_{n}(F)$ is a return sequence for $T$, which is unique up to asymptotic equivalence.

Next we will estimate the return sequence $a_{n}(F)$.

Lemma 4.6. Let $\left(\Delta, \mathcal{B}(\Delta), F, m^{\prime}\right)$ be a Young tower with base $\Delta_{0}$ (for some nonsingular dynamical system $(X, \mathcal{B}, m, T))$ and suppose that $m_{0}(\{R>n\}) \propto n^{-\beta}$ for some $0<\beta \leq 1$. Then $w_{n}(F) \in \mathcal{R}_{-\beta}$ and $a_{n}(F) \in \mathcal{R}_{\beta}$.

Proof. Let $\varphi_{\Delta_{0}}(x):=\min \left\{n \geq 1: F^{n}(x) \in \Delta_{0}\right\}$ be the first return time function of $F$ to $\Delta_{0}$ and observe that $\varphi_{\Delta_{0}}(x)=R(x)$ for all $x \in \Delta_{0}$. From Lemma 4.4 we know that $F$ admits an invariant measure $\nu$ with $\left.\nu\right|_{\Delta_{0}}=\nu_{0}$ where $\nu_{0} \sim m_{0}$ is the invariant for measure for $F^{\varphi_{\Delta_{0}}}$. Thus, there exist $c>0$ such that,

$$
\begin{aligned}
w_{n}\left(\Delta_{0}\right) & =\nu\left(\cup_{k=0}^{n-1} F^{-k} \Delta_{0}\right)=\sum_{k=0}^{n-1} \nu\left(\Delta_{0} \cap\left\{\varphi_{\Delta_{0}}>k\right\}\right) \\
& =c \sum_{k=0}^{n-1} \nu_{0}(\{R>k\}) \propto \sum_{k=0}^{n-1} m_{0}(\{R>k\}) \in \mathcal{R}_{1-\beta},
\end{aligned}
$$

where $\sum_{k=0}^{n-1} m_{0}(\{R>k\}) \in \mathcal{R}_{1-\beta}$ by Karamata's Theorem (part 1$)$ if $0<\beta<1$ and $\sum_{k=0}^{n-1} m_{0}(\{R>k\})=(\log n) l(n)$ if $\beta=1$, where $l(n) \in \mathcal{R}_{0}$.

From Lemma 4.4 we know that $\Delta_{0}$ is a Darling-Kac set for $F$. Therefore, $w_{n}(F) \in$ $\mathcal{R}_{-\beta}$ by Theorem 3.8.3 in [1]. Furthermore, $a_{n}(F) \in \mathcal{R}_{\beta}$ by Lemma 2.4.

We can now conclude 
Proof of Corollary 2.6. Since $a_{n}(F) \in \mathcal{R}_{\beta}$ with $\beta \in[0,1]$, i1) follows by Proposition 2.5.

i2) follows by the same argument since $a_{n}(F)$ is also a return sequence for $T$.

Proof of Corollary 2.7. The conditions of Theorem 2.1 together with Lemma 4.5 and Lemma 4.6 implies that $w_{n}(T) \propto w_{n}\left(F_{1}\right) \in \mathcal{R}_{-\beta}$ as $n \rightarrow \infty$. By the same argument for $F_{2}, w_{n}(T) \propto w_{n}\left(F_{2}\right)=\sum_{k=0}^{n-1} m_{0_{2}}\left(\left\{R_{2}>k\right\}\right)$. Thus,

$$
\sum_{k=0}^{n-1} m_{0_{2}}\left(\left\{R_{2}>k\right\}\right) \propto \sum_{k=0}^{n-1} m_{0_{1}}\left(\left\{R_{1}>k\right\}\right)
$$

and the conclusion follows immediately by Proposition 4.1 and Proposition 4.2.

Remark 4.7. We notice that from the above proof we have that

$$
w_{n}(T) \propto \sum_{k>n} m_{0_{1}}\left(\left\{R_{1}>k\right\}\right) \propto \sum_{k>n} m_{0_{2}}\left(\left\{R_{2}>k\right\}\right)
$$

independently of the assumption of the regular variation of the tail sequences. The fact that $\sum_{k>n} m_{0_{1}}\left(\left\{R_{1}>k\right\}\right) \propto \sum_{k>n} m_{0_{2}}\left(\left\{R_{2}>k\right\}\right)$ trivially holds in the probability case $\beta>1, \gamma>1$, since the two tail sequences are summable. However, as it is obvious from the proof, in this case, the conclusion $m_{0_{1}}\left(\left\{R_{1}>k\right\}\right) \propto m_{0_{2}}\left(\left\{R_{2}>k\right\}\right)$ does not follow.

\section{Infinite oscillation at one of the indifferent fixed points}

As mentioned in Section 2.2, we conclude with one example that illustrates the use of Corollary 2.6 and as such, the usefulness of modelling infinite measure preserving transformations via YTs. Darling Kac-like theorems were proved for increasingly general systems. The version in Aaronson's book [1, Theorem 3.6.4. and Corollary 3.7.3] requires that $T$ is p.d.e. and the return sequence $\left(a_{n}\right)$ is regularly varying, which is in general difficult to check. In [20], the p.d.e. property was established for non-uniformly expanding interval maps with indifferent fixed points (AFN-maps). Thaler and Zweimüller [16, Theorem 1] then replaced the regular variation of the return sequence by that of the wandering rate $w_{n}(T)$ (which is easier to check) together with the requirement that

$$
h_{N}:=\frac{1}{w_{N}(Y)} \sum_{n=0}^{N-1} \hat{T} 1_{\{x \in X \backslash Y: R(x)=n\}}
$$

be convergent ${ }^{4}$ uniformly on the Darling Kac set $Y$. In [23, Theorem 2.1] finally, Zweimüller weakened the convergence requirement to $\left(h_{N}\right)_{N \in \mathbb{N}}$ being precompact in $L^{\infty}(\mu)$. This enabled him to treat maps with multiple indifferent fixed points where the strength of the one mutually majorizes and minorizes the strength of another, depending on the distance to these fixed points.

The example we consider below is a particular case of the somewhat abstract Example 7.1 in [23] which Zweimüller gave to show the advantage of [23] over [16]. The regular variation of the wandering rate $w_{n}(T)$ with some index $\beta \in[0,1]$ in Example 7.1 of [23] is explicitly given, which allows an immediate application of [23,

\footnotetext{
${ }^{4}$ with a uniformly sweeping limit
} 
Theorem 2.1]. In contrast, the example below does not provide an explicit form of $w_{n}(T)$. By considering an appropriate YT extension for $T$, we simultaneously obtain the p.d.e. property, regular variation of the return sequence $a_{n}$, and our DK version Corollary 2.6.

Let $T:[0,1] \rightarrow[0,1]$ be a map with two indifferent fixed points at 0 and 1 with $T([0,1 / 2))=(0,1), T([1 / 2,1))=(0,1)$ such that for $p>1$,

$$
T(x)= \begin{cases}x+x^{1+p} l(1 / x)+o\left(x^{1+p} l(1 / x)\right) & \text { as } x \rightarrow 0 \\ x+(1-x)^{1+p}+o\left((1-x)^{1+p}\right) & \text { as } x \rightarrow 1\end{cases}
$$

where $l(t)=\exp \left[(\log t)^{1 / 3} \cos (\log t)^{1 / 3}\right], t>0$, is in $\mathcal{R}_{0}$ with infinite oscillation, i.e.,

$$
\lim _{t \rightarrow \infty} \inf l(t)=0 \text { and } \lim _{t \rightarrow \infty} \sup l(t)=\infty .
$$

Take $0<\cdots<x_{2}<x_{1}<x_{0}=1 / 2=x_{0}^{\prime}<x_{1}^{\prime}<x_{2}^{\prime}<\cdots<1$ such that $T\left(x_{n}\right)=x_{n-1}$ and $T\left(x_{n}^{\prime}\right)=x_{n-1}^{\prime}$ for all $n \geq 1$. Let $I_{n}=\left(x_{n+1}, x_{n}\right), I_{n}^{\prime}=\left(x_{n}^{\prime}, x_{n+1}^{\prime}\right)$ for $n \geq 0$ and let $\left.R\right|_{I_{n} \cup I_{n}^{\prime}}:=n+1$. By (16), the asymptotic of $\left\{x_{n}\right\},\left\{x_{n}\right\}^{\prime}$ are as follows:

$$
x_{n} \sim n^{-1 / p} l(n) \quad \text { and } \quad 1-x_{n}^{\prime} \sim n^{-1 / p} .
$$

Thus the tail of the inducing scheme is

$$
m(\{R>n\})=\sum_{k \geq n} m\left(I_{k} \cup I_{k}^{\prime}\right) \sim n^{-p}(l(n)+1) .
$$

Construct a Young tower with $\Delta_{0}=(0,1)$, partition $\left\{\Delta_{0, i}\right\}=\left\{I_{i}\right\} \cup\left\{I_{i}^{\prime}\right\}$ and return time $R$ as indicated. Then $T^{R}\left(I_{n}\right)=\left(\frac{1}{2}, 1\right)$ and $T^{R}\left(I_{n}^{\prime}\right)=\left(0, \frac{1}{2}\right)$ for each $n$, so (YT2') of Section 4.1 is satisfied.

The distortion condition (YT6) follows by the argument of Lemma 5 in [17]. Also, notice that g.c.d $\left(R_{i}\right)=1$, (so (YT7) holds) and thus, the good properties for $F$ follow immediately by Lemma 4.4 .

To check the conditions of Theorem 2.1, we just need to observe that inducing w.r.t. first returns on any $\Delta_{0, i}$ gives rise to a finite $\left(T^{R}\right)^{\varphi_{\Delta_{0, i}}^{R} \text {-invariant measure, }}$ which proves the integrability of $\varphi_{\Delta_{0, i}^{R}}$ on $\Delta_{0, i}$. Thus, if $\tau$ and $\rho$ are given by $T^{\rho}=\left(T^{R}\right)_{\Delta_{0, i}}=\left(T_{\Delta_{0, i}}\right)^{\tau}$, the integrability of $\tau$ follows immediately since $\{\tau>$ $n\}=\left\{\rho>\varphi_{\Delta_{0, i}^{R}}\right\}$ by Fact 3.3 (see also [21] for details). Because we also have $m(\{R>n\}) \in \mathcal{R}_{-1 / p}$, Corollary 2.6 immediately applies. In particular, Lemma 2.4 gives the exact form of $a_{n}(T)$.

\section{References}

[1] J. Aaronson, An Introduction to Infinite Ergodic Theory, Mathematical Surveys and Monographs, 50 American Mathematical Society, Providence, (1997)

[2] J. Aaronson, M. Denker, M. Urbanski, Ergodic theory for Markov fibred systems and parabolic rational maps, Trans. AMS. 337 (1993), 495-548.

[3] J. Aaronson, M. Thaler, R. Zweimüller, Occupation times of sets of infinite measure for ergodic transformations, Ergod. Th. \& Dynam. Sys. 25 (2005), 959-976.

[4] J. Al-Khal, H. Bruin, M. Jakobson New examples of S-unimodal maps with sigma-finite absolutely continuous invariant measures, Disc. Cont. Dyn. Sys. 22 (2008), 35-61. 
[5] N. Bingham, C. Goldie, J. Teugels, Regular variation, Cambridge. University Press (1989)

[6] H. Bruin, Topological conditions for the existence of invariant measures for unimodal maps, Ergod. Th. \& Dynam. Sys. 14 (1994), 433-451.

[7] H. Bruin, Induced maps, Markov extensions and invariant measures in onedimensional dynamics, Commun. Math. Phys. 168 (1995) 571-580.

[8] J. Buzzi, V. Maume-Deschamps, Decay of correlations on towers with nonHölder Jacobian and non-exponential return time, Discrete Contin. Dyn. Syst. 12 (2005), 639-656.

[9] P. Collet, P. Ferrero, Some limit ratio theorem related to a real endomorphism in case of a neutral fixed point, Annales de l'institut Henri Poincaré (A) Physique théorique 52 (1990), 283-301.

[10] W. de Melo and S. van Strien, One-Dimensional Dynamics, Springer, Berlin Heidelberg New York, (1993).

[11] S. Isola, On the spectrum of Farey and Gauss maps, Nonlinearity 15 (2002), 1521-1539.

[12] S. Isola, Renewal sequences and intermittency, Journal of Statistical Physics 97 (1999), 263-280.

[13] M. Campanino, S. Isola, Infinite invariant measures for non uniformly expanding transformations of $[0,1]$; weak law of large numbers with anomalous scaling, Forum Math. 8 (1996), 71-92.

[14] M. Thaler, Transformations on [0,1] with infinite invariant measures, Isr. J. Math. 46 (1983), 67-96.

[15] M. Thaler, A limit theorem for the Perron-Frobenius operator of transformations on $[0,1]$ with indifferent fixed points, Isr. J. Math. 91 (1995), 111-127.

[16] M. Thaler, R. Zweimüller, Distributional limit theorems in infinite ergodic theory, Probability Theory and Related Fields 135 (2006), 15-52.

[17] L.-S. Young, Statistical properties of dynamical systems with some hyperbolicity, Annals of Math. 147 (1998), 585-650.

[18] L.-S. Young, Recurrence times and rates of mixing, Israel. J. Math. 110 (1999) $153-188$.

[19] R. Zweimüller, Ergodic structure and invariant densities of non-Markovian interval maps with indifferent fixed points, Nonlinearity 11 (1998), 1263-1276.

[20] R. Zweimüller, Ergodic properties of infinite measure preserving interval maps with indifferent fixed points, Ergod. Th. \& Dynam. Sys. 20 (2000), 1519-1549.

[21] R. Zweimüller, Invariant measures for generalized induced transformations, Proceedings AMS. 133 (2005), 2283-2295.

[22] R. Zweimüller, Addendum to "Invariant measures for generalized induced transformations", Proceedings AMS. 138 (2005), 2283-2295.

[23] R. Zweimüller, Infinite measure preserving transformations with compact first regeneration, Journal d'Analyse Mathematique, 103 (2007), 93-131.

[24] R. Zweimüller, Waiting for long excursions and close visits to neutral fixed points of null-recurrent ergodic maps, Fundamenta Math. 198 (2008), 125138. 
[25] R. Zweimüller, Measure preserving transformations similar to Markov shifts, Israel J. Math. to appear .

Department of Mathematics

University of Surrey

Guildford, Surrey, GU2 7XH

UK

h.bruin@surrey.ac.uk

http://personal.maths. surrey.ac.uk/st/H.Bruin/

Department of Mathematics

University of Houston

Houston TX 77204-3008

USA

nicol@math.uh.edu

http://www.math.uh.edu/

Department of Mathematics

University of Surrey

Guildford, Surrey, GU2 7XH

UK

d.terhesiu@surrey.ac.uk 